Recibido: 23.06.2017. Aceptado: 02.10.2017.

\title{
EL IMPACTO DE LAS TECNOLOGÍAS DE LA INFORMACIÓN Y DE LA COMUNICACIÓN EN LA SALUD DE LOS TRABAJADORES: EL TECNOESTRÉS
}

\author{
THE IMPACT OF INFORMATION AND COMMUNICATION \\ TECHNTECHNOLOGIES ON WORKERS' HEALTH: \\ TECHNOESTRÉS
}

\section{LUCÍA ARAGÜEZ VALENZUELA ${ }^{1}$}

Universidad de Málaga

\footnotetext{
${ }^{1}$ Personal Investigador en Formación. Departamento de Derecho del Trabajo y de la Seguridad Social. Universidad de Málaga. Facultad de Derecho. Bulevar Louis Pasteur, 26, 29071, Málaga (Campus Teatinos).
} 


\title{
RESUMEN
}

El uso de las tecnologías se ha introducido con rapidez en la sociedad, formando parte irreversiblemente de nuestras vidas cotidianas. En el mundo laboral, su uso -y abusoha generado diversas formas de trabajo y de organización empresarial, primando la prestación de servicios mediante la utilización de sistemas informáticos.

Sin embargo, las tecnologías pueden crear unas exigencias para el trabajador que, en ocasiones, es incapaz de asumir -bien sea por falta de formación, adaptación o por el uso inadecuado de las mismas-, dando lugar a nuevos riesgos psicosociales en el trabajo -como es el tecnoestrés-.

Este estudio tratará de analizar la repercusión de las tecnologías en el mundo laboral, teniendo a su vez en cuenta las innumerables ventajas laborales promovidas por su incorporación y manteniendo la idea de que es necesario hacer un uso razonable de las mismas para evitar circunstancias perjudiciales en la salud de los trabajadores.

PALABRAS CLAVE: tecnoestrés, trabajadores, riesgo psicosocial, tecnologías.

\begin{abstract}
The use of technologies has been introduced rapidly in society, forming an irreversible part of our daily lives. In the labor world, its use - and abuse - has generated various forms of work and business organization, with the primary provision of services through the use of computer systems.

However, the use of Technology may create requirements that are often unable to assume by some employees, either due to lack of training, lack of adaptation or misuse of them. This creates a situation of psychosocial risk, the so-called technostress, which directly affects their health and undoubtedly makes difficult to balance working life with private life.

This study will analyze the impact of technologies in the world of work, taking into account the innumerable labour advantages promoted by its incorporation and maintaining the idea that it is necessary to make a reasonable use of them to avoid detrimental circumstances in The health of workers.
\end{abstract}

KEYWORDS: technostress, workers, psychosocial risk, technologies. 
SUMARIO

\section{INTRODUCCIÓN}

II. LA APARICIÓN DE NUEVOS RIESGOS PSICOSOCIALES EN EL TRABAJO: EL TECNOESTRÉS

III. POSIBLES MEDIDAS PREVENTIVAS DEL TECNOESTRÉS

IV. AUSENCIA DE REGULACIÓN ESPECÍFICA Y ANÁLISIS DEL DERECHO A DESCONECTARSE

V. CONCLUSIONES 


\section{INTRODUCCIÓN}

El uso de las tecnologías de la información y de la comunicación -también conocidas como $\mathrm{TIC}^{2}$ - forman parte de la vida cotidiana de forma indeleble.El paradigma digital está imponiendo transformaciones abruptas y disruptivas en todos los escenarios de la vida en sociedad; sin embargo, la intensidad de tales cambios se aumenta entre los escenarios y actores protagonistas de dichas modificaciones ${ }^{3}$ : los trabajadores.

Las TIC han evolucionado de forma vertiginosa ${ }^{4}$ en el mundo del trabajo, pasando de ser utilizadas por un pequeño número de trabajadores, a generalizarse su uso; tanto es así que desde mediados de los noventa hasta la actualidad, las tecnologías han abarcado ámbitos sociales, culturales, económicos, docentes $\mathrm{y}$, por supuesto, laborales ${ }^{5}$. Concretamente, la Unión Europea (UE) acuñó el término Sociedad de la Información a partir del Informe BANGEMANN como una estrategia de incorporación de las nuevas tecnologías de la información y de la comunicación en la Europa del futuro ${ }^{6}$, fomentando la utilización de dichos medios informáticos como un proceso de evolución.

Debido a estos nuevos modelos con implicaciones digitales y propensos a la incorporación de las tecnologías en casi todos los escenarios de la vida en sociedad, el mundo del trabajo se concierne de forma distinta, creando estructuras productivas flexibles, modificando la propia relación laboral; e incluso, llegando a cuestionar conceptos clásicos del derecho del trabajo. Así, cada vez un mayor número de empresas desarrollan su actividad laboral mediante la utilización de plataformas virtuales o a través de la incorporación de las tecnologías de la información y la comunicación en el trabajo. Este protagonismo tecnológico se debe a una forma empresarial de reducir costes, mejorar la efectividad y obtener beneficios económicos para ser consideradas en el mercado como empresas más competitivas ${ }^{7}$

\footnotetext{
${ }^{2}$ Se consideran TIC's al conjunto de tecnologías, tales como: software, soportes, canales, herramientas, etc., que permiten al usuario acceder, almacenar, transmitir y procesar la información.

${ }^{3}$ Alemán Páez, F.; El derecho de desconexión digital. Una aproximación conceptual, crítica y contextualizadora al hilo de la LOU Travail $n^{\circ}$ 2016-1088. Ponencia del IV Congreso Iberoamericano y Europeo sobre Nuevas formas de trabajo y su regulación. Incidencia de las migraciones laborales en la seguridad social. Cuenca. 2016.

${ }^{4}$ Manzano Santamaría, N.; Las tecnologías de la comunicación y la información (TIC's) y las nuevas formas de organización del trabajo: factores psicosociales de riesgo. Anuario Internacional sobre prevención de Riesgos Psicosociales y calidad de vida en el trabajo. 2016. Pág. 29-55. Disponible en el siguiente enlace: http://www.ugt.es/Publicaciones/Anuario2016\%20WEB.pdf

${ }^{5}$ El Plan de Acción e Europe 2002 e INFO XXI fueron programas impulsadores del proceso de creación de una cibersociedad tecnificada con objetivos tales como: el estímulo del uso de Internet de forma generalizada, la inversión en personas y en su formación en lo que al uso de las tecnologías se refiere, la mejora de Internet haciéndolo más rápido y seguro, entre otros

${ }^{6}$ Saiz Álvarez, J. M.; Nuevas tecnologías y mercado de trabajo: situación actual de la externalización de servicios. Ponencia para el I Congreso Internacional sobre Tecnología Documental y del Conocimiento por la AHDI. Madrid. 2004. Disponible en el siguiente enlace: http://eprints.rclis.org/5480/1/Madrid15.pdf

${ }^{7} \mathrm{~A}$ su vez, ha surgido un nuevo concepto de práctica laboral, el eWork mediante la incorporación de las TIC's en el desempeño del trabajo gracias a la colaboración de equipos de trabajo móviles virtuales. Espacios donde una red internacional de emprendedores y trabajadores pueden relacionarse, colaborar, trabajar y aprender.
}

e-Revista Internacional de la Protección Social, ISNN 2445-3269. 2017, Vol. II, No 2 
Esta situación cambiante en las condiciones de trabajo, ha dado lugar a que los trabajadores tengan que readaptarse al uso de las tecnologías y también formarse en esta materia, mediante el aprendizaje de estos soportes electrónicos, como por ejemplo: el uso del correo electrónico, la firma electrónica, de Internet, la posible vigilancia y control por parte del empresario a la hora de desarrollar su actividad laboral ()cámaras de videovigilancia, sistemas de escucha, GPS, redes sociales, etc.), el tratamiento automatizado de datos, entre otros.

Ante esta nueva realidad laboral es necesario, de un lado, que los trabajadores tengan una actitud predispuesta al cambio mediante la adaptación de las tecnologías a la reestructuración de la profesión, logrando un enfoque integrado que asocie la introducción de nuevas tecnologías con la educación, el aprendizaje y los cambios organizacionales en la empresa; y de otro lado, que el legislador vea la necesidad de regular la materia -en cualquiera de sus formas ${ }^{8}$ - para evitar abusos en los derechos básicos y fundamentales de los trabajadores.

Aquellos trabajadores que se han adaptado a la perfección a los cambios tecnológicos en el puesto de trabajo, son considerados como: empleados 3.0, los cuales tratan de conseguir el máximo beneficio para la empresa mediante la utilización de los medios tecnológicos de forma equilibrada y adecuada, maximizando los recursos que las TIC ofrecen al trabajo (la comunicación sincrónica, el acceso, la obtención y la utilización de información, la movilidad de contenidos, el abaratamiento físico laboral, espacios de coworking ${ }^{9}$, etc.). Sin embargo, en la mayoría de los casos, los trabajadores tienen una mala adaptación al uso de las tecnologías -bien sea por defecto o bien por exceso-, dando lugar a situaciones estresantes en su puesto de trabajo.

\section{LA APARICIÓN DE NUEVOS RIESGOS PSICOSOCIALES EN EL TRABAJO: EL TECNOESTRÉS}

Los riesgos psicosociales en el ámbito laboral ${ }^{10}$ hacen referencia a aquellas circunstancias perjudiciales en la salud mental, física y social de los trabajadores, ocasionados por las condiciones de trabajo y por los factores organizacionales y relacionales susceptibles de interactuar con el funcionamiento mental.

\footnotetext{
${ }^{8}$ Regulación legal, reglamentaria, por negociación colectiva, por contrato de trabajo individual, o por convenio.

10 Pueden consultarse en la Nota Técnica de Prevención 926 del Instituto Nacional de Seguridad e Higiene en el Trabajo (INSHT, 2012) y en el documento del European Framework forPsyscho social Risk Management (PRIMA-EF, 2008).
}

e-Revista Internacional de la Protección Social, ISNN 2445-3269. 2017, Vol. II, No 2 


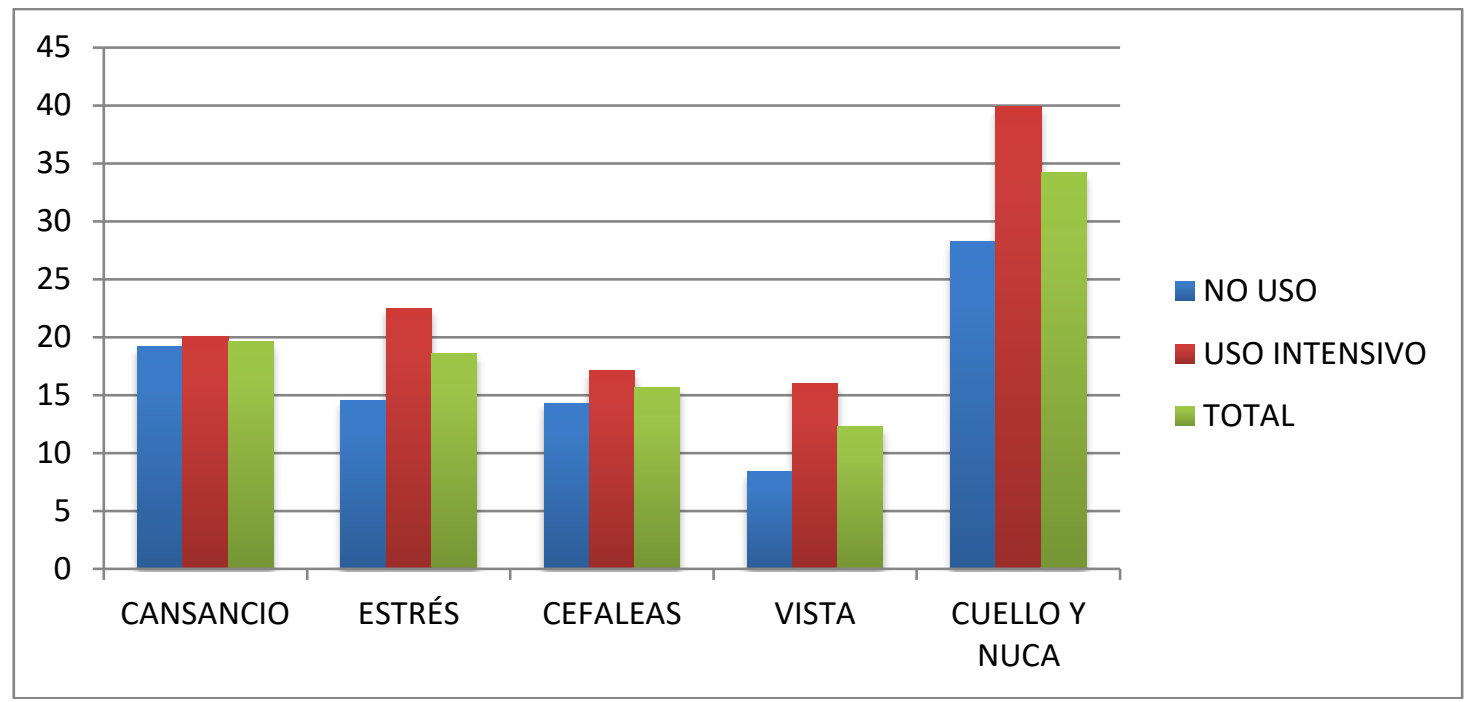

Fuente: Anuario Internacional sobre prevención de Riesgos Psicosociales y calidad de vida en el trabajo 2016

Actualmente, la Comisión Nacional de Seguridad y Salud en el Trabajo (CNSST) ha considerado estas nuevas $-\mathrm{y}$ no tan nuevas- enfermedades de trabajo como causas motivadoras de incapacidad laboral en los trabajadores. Concretamente en este estudio, vamos a analizar el estrés laboral ${ }^{11}$-o más bien el tecnoestrés- como trastorno protagonista en la actualidad tras la incorporación de las tecnologías de la información y de la comunicación en las condiciones de trabajo.

Concretamente, la flexibilidad laboral, la deslocalización, el aumento de competitividad en el mercado y el uso de las TIC en el mercado laboral, están propiciando la generación de riesgos emergentes de carácter psicosocial: ${ }^{12}$

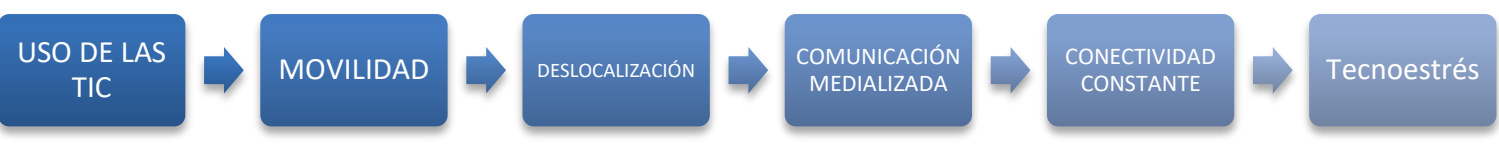

En la actualidad, existen dos tipos de trastornos derivados por la incorporación inadecuada de las TIC en el trabajo: la tecnofobia y la tecnoadicción, siendo ambas consideradas como elementos motivadores del estrés en el trabajo.

\footnotetext{
${ }^{11} \mathrm{El}$ estrés es una respuesta del organismo que puede afectar gravemente a la salud, el bienestar y la calidad de vida del que lo padece y de los que le rodean. Tiene consecuencias psicológicas y fisiológicas. En efecto es un factor de riesgo de tres de las principales causa de muerte de las sociedades actuales: cáncer, enfermedades cardiacas y accidentes cerebro vasculares.

${ }^{12}$ Manzano Santamaría, N.; Las tecnologías de la comunicación y la información (TIC's) y las nuevas formas de organización del trabajo: factores psicosociales...op. cit.
} 
De un lado, la tecnofobia ${ }^{13}$ es considerada como aquella enfermedad en la que los trabajadores sienten un rechazo frontal al uso de las nuevas tecnologías en el trabajo, es decir, se consideran tecnófobos aquellas personas que presentan una actitud general en contra a las nuevas tecnologías. Los primeros tecnófobos reconocidos por la historia fueron los luditas o ludistas que conformaron un violento movimiento contra la revolución industrial que surgió en Inglaterra a comienzos del siglo XIX ${ }^{14}$. Los ludistas eran antiguos artesanos tejedores que veían amenazada su subsistencia por los telares mecánicos y por la mano de obra barata que se contrataba. Sin embargo, hoy en día el concepto de tecnófobo ha cambiado, pero continúa manteniendo su esencia en lo que respecta al rechazo del elemento tecnológico tanto en su vida cotidiana como en el trabajo.

Los grupos más sensibles a este tipo de trastornos son generalmente los trabajadores de edad media o avanzada (a partir de cuarenta años de edad). El motivo fundamental consiste en que les resulta bastante complejo reaprender una nueva forma de trabajo a través de sistemas electrónicos o informáticos. Sin embargo, es cierto que dicho colectivo-denominado: trabajadores maduros- no únicamente se inmiscuyen en este tipo de trastorno, sino que se puede ampliar también a trabajadores de diferentes edades que sienten miedo a lo desconocido y a sentirse amenazados o atacados por cualquier tipo de innovación tecnológica ${ }^{15}$.

El trastorno de la tecnofilia -o el miedo a las tecnologías- encuentra su razón de ser, de entre otros muchos escenarios, a la escasa formación de los trabajadores en la materia, fundamentalmente de aquellos que no se encuentran relacionados con las TIC en su vida cotidiana, haciéndose muy complicada la adaptación mental a su nuevo puesto de trabajo.

De otro lado, nos podemos encontrar con trabajadores que mantienen una adicción al uso de las nuevas tecnologías y no únicamente en su vida cotidiana, sino también en su propio puesto de trabajo. Antes de adentrarnos en esto, debemos tener claro una serie de conceptos. La OMS (Organización Mundial de la Salud) en 1989 definió la adicción en términos de dependencia, esto es, el uso de algo debido al alivio, confort o estimulación que se consigue con su utilización. Según ECHEBURÚA y CORRAL ${ }^{16}$ cualquier

\footnotetext{
${ }^{13}$ Elizabeth Luque, L.; Tecnofobia: estudio sobre la ansiedad ante y la aversión hacia las herramientas de tecnología digital, en sujetos adultos argentinos. Interpsiquis. 2006. Disponible en el siguiente enlace: http://www.psiquiatria.com/bibliopsiquis/assetstore/13/07/22/13072252889899633160918553489992220 8778. Según la doctora, la tecnofobia se basa en los dos siguientes aspectos: "1) La incorporación y el incremento de herramientas de tecnologías digitales (HTD) en la vida cotidiana caracterizado básicamente por la ansiedad antes las HD y los comportamientos de aversión consecuentes. 2) los tecnofóbicos evitan, en la medida de lo posible, la interacción con las HTD pero cuando se ven forzados al uso de éstas por sus actividades laborales o académicas, tardan más tiempo, cometen más errores y se desempeñan más pobremente que los no-fóbicos"

${ }^{14}$ Martínez Selva, J.M.;Tecnoestrés: ansiedad y adaptación a las nuevas tecnologías en la era digitalGrupo Planeta. 2011.Pág. 15.

15 Sócrates, según narra Platón en Fedro, desconfiaba de la escritura porque podría provocar en las personas la merca en la facultad de la memoria.

16 Madrid López, R. I.;Adicción a internet: Conceptualización y Mecanismos explicativos.PSIQUIATRIA.COM. 2000. Pág. 2. Véase en el siguiente enlace: https://www.psiquiatria.com/revistas/index.php/psiquiatriacom/article/viewFile/542/520.
} 
conducta normal placentera es susceptible de convertirse en un comportamiento adictivo.

Cuando un trabajador mantiene cierta adicción a la utilización de las tecnologías de la información y de la comunicación en su puesto de trabajo, se considera que tiene un determinado trastorno: la tecnofilia ${ }^{17}$, que no es más que aquella clásica adicción pero circunscrita al ámbito de las tecnologías de la información y de la comunicación. Los tecnófobos por excelencia son los jóvenes, es decir, aquellos trabajadores que han nacido a partir de 1980 los cuales se encuentran intrínsecamente relacionados con la utilización de tecnologías de la información y de la comunicación, siendo considerados propensos a generar tecno-adicciones por el manejo con facilidad de dichos medios.

En el ámbito concreto de las tecnologías, su uso -y abuso- puede llevar a desarrollar un patrón conductual adictivo a las mismas que, si directamente se relaciona con el trabajo, puede entenderse adicción a ambas (tecnologías y trabajo). El patrón adictivo en el caso de los adictos a Internet se denomina: desorden de adicción a Internet -Internet Addiction Disorder (IAD)-, uso compulsivo de Internet, o uso patológico de Internet Pathological Internet Use (PIU)-.

En el caso de la tecnofilia, y a diferencia de la tecnofobia, los trabajadores no han tenido que aprender la utilización de dichos medios en el trabajo, sino que forma parte de su vida cotidiana. Tal es el grado de utilización compulsiva de dichos medios que incluso, en ocasiones, les dificulta la concepción de trabajo sin la utilización de dichos medios electrónicos en el desempeño de su actividad.

Estos dos extremos (tecnofobia y tecnofilia) tienen indiscutiblemente efectos negativos para la salud de los trabajadores y también para la posible conciliación de su vida personal, familiar y laboral. De un lado, la tecnofobia puede generar un riesgo psicosocial denominado: tecnoestrés, que no es más que un tipo específico de estrés laboral promovido por la falta de capacidad de adaptación a las nuevas tecnologías en el trabajo, entre otros aspectos. De otro lado, también puede generar situaciones tecnoestresantes e impidiendo el disfrute pleno de la vida personal y familiar del trabajador por estar constantemente conectado a su trabajo. Así, el trabajo no termina cuando acaba la jornada laboral, sino que resulta una característica usual que los adictos se lleven trabajo a casa, trabajen los fines de semana, durante las vacaciones e incluso estando enfermos ${ }^{18}$.

Muchas son las clasificaciones posibles de los factores de riesgos psicosociales que existen en la actualidad. Concretamente en este estudio se tendrá en cuenta la clasificación del Colegio de Expertos sobre el Seguimiento de los Riesgos Psicosociales en el Trabajo de Francia, el cual señala:

A. Intensidad del trabajo y tiempo de trabajo. La intensidad del trabajo es una noción muy utilizada pero difícil de definir. Se podría considerar la

\footnotetext{
${ }^{17} \mathrm{La}$ tecnofilia se trata de afición a la tecnología, existiendo distintos grados de tecnofilia dependiendo de la atracción o dependencia que el tecnófilo tenga hacia las tecnologías.

${ }^{18}$ Salanova Soria, M.,; Nuevas tecnologías y nuevos riesgos psicosociales en el trabajo, RevistaDigital de Seguridad y Salud en el Trabajo, N 1, 2007. Pág.14.
} 
intensidad como aquella complejidad del trabajo digital, al ser menos homogéneo ${ }^{19}$.

La utilización de las tecnologías es un factor que puede dar lugar a un aumento en la intensidad en el trabajo, principalmente con respecto a su duración, organización del tiempo, extensión de la disponibilidad horaria del trabajador, existencia casi permanente del teletrabajo -además del trabajo normal-, vínculo casi permanente con la empresa, horarios irregulares, mayores exigencias emocionales, etc. (tecnofilia).

B. Exigencias emocionales: sentimiento de miedo o rechazo a la incorporación de las nuevas tecnologías de la información y de la comunicación en las condiciones de su puesto de trabajo. Lo cual, además, puede desencadenar un menor contacto interpersonal con sus compañeros, clientes, empresario, y relación con el público en general (tecnofobia).

C. Inseguridad en el trabajo: inseguridad a la hora de efectuar el desarrollo de la actividad laboral por la incorporación de las tecnologías en el puesto de trabajo; inseguridad a su vez en el sentido de no verse compensado las horas de trabajo efectivo incluso fuera de su horario laboral; e igualmente inseguridad con respecto a la escasa formación e inadaptación a los cambios en la propia persona del trabajador.

La clave para evitar estos riesgos estriba en la generalización de una sociedad del aprendizaje ${ }^{20}$ que suponga un cambio de cultura social tendente a la digitalización, y ocasionando a los trabajadores un continuo esfuerzo adaptativo respecto a los códigos y los ritmos del "tecnoglobalismo".

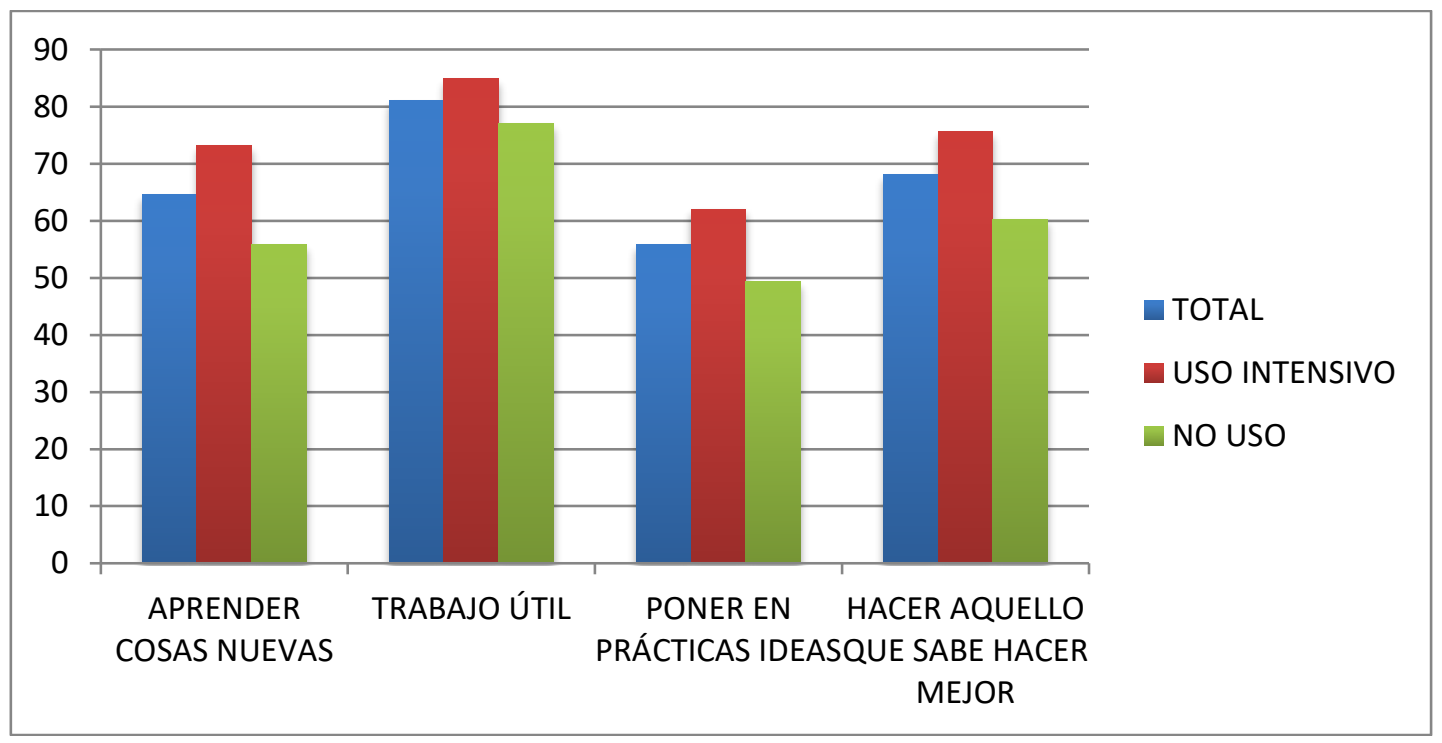

\footnotetext{
${ }^{19}$ Por ejemplo puede hacer que varíe un puesto de trabajo a otro, o incluso dentro del mismo puesto de trabajo que se produzcan modificaciones.

${ }^{20}$ Marina Torres, J.A.; Despertad al diplodocus. Una conspiración educativa para transformar la escuela y todo lo demás. Ed. Ariel, Barcelona, 2015.
}

e-Revista Internacional de la Protección Social, ISNN 2445-3269. 2017, Vol. II, No 2 
Fuente: Anuario Internacional sobre prevención de Riesgos Psicosociales y calidad de vida en el trabajo 2016

Las TIC imponen conductas obligacionales, tanto de auto-exploración como de heteroexploración y, en general, suponen más trabajo fuera del horario estrictamente laboral al diluir los códigos de espacio-tiempo durante la jornada de trabajo, pues normalizan su uso durante las veinticuatro horas a escala planetaria; y además, las fórmulas de "trabajo a proyecto", "lavoro agile" o "trabajo a cliente", o la "autonomización del empleo"21.

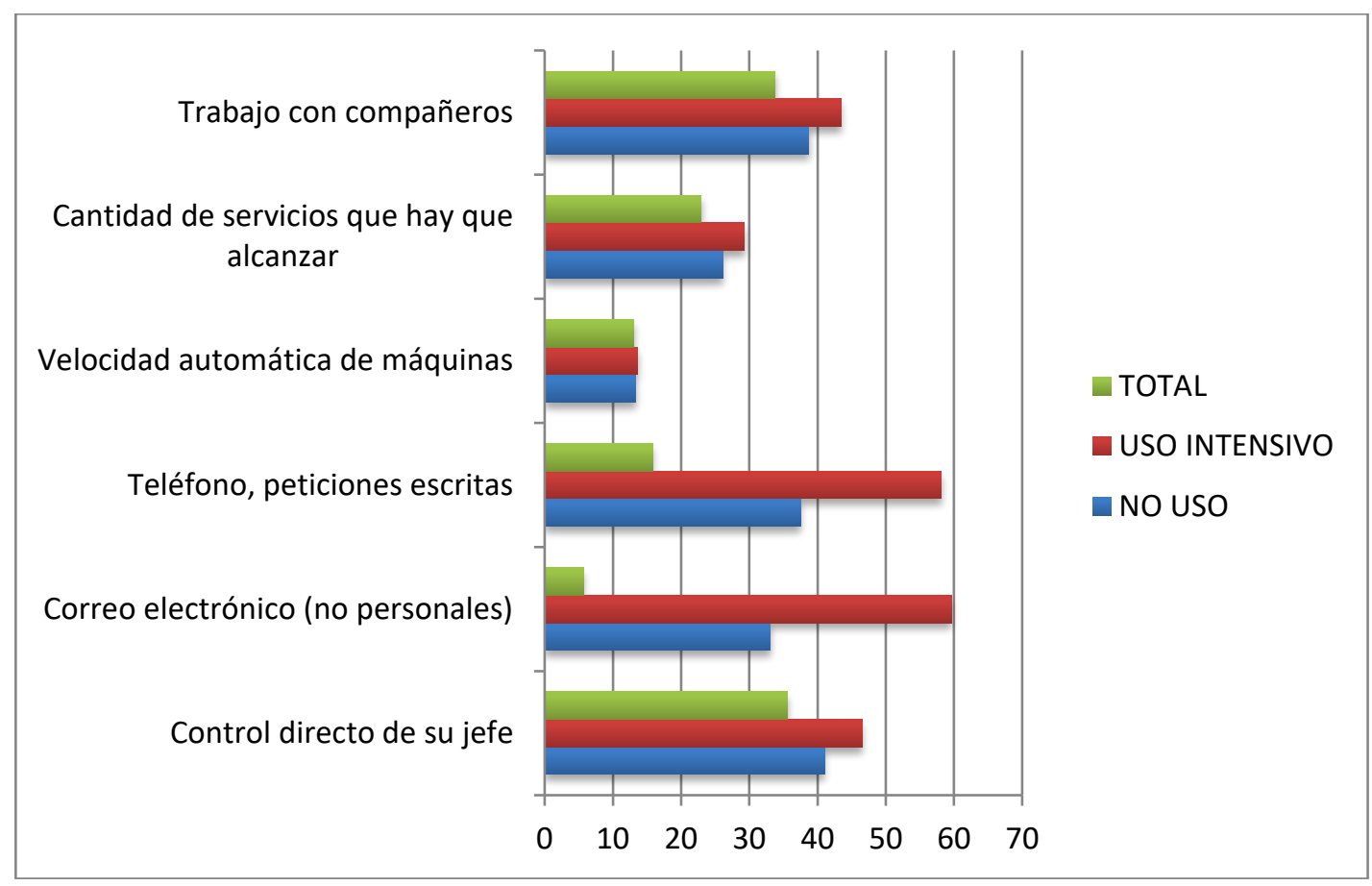

Fuente: Anuario Internacional sobre prevención de Riesgos Psicosociales y calidad de vida en el trabajo 2016

Paralelamente el escenario descrito, también se proyecta un amplio abanico de problematizaciones paralelas. "Cibercansancio", "tecno-fobia", "tecno-filia", "tecnoadicción", "tecno-ansiedad", "habeas data", "esquirolaje tecnológico", "cibervigilancia", "autodeterminación informativa", "expectativas de confidencialidad", o el propio "derecho de desconexión", que conforman y engloban todos ellos un mapa deletéreo $^{22}$ de las TIC. En este sentido, BRILLHART distinguió cuatro situaciones distintas que ocasionaban el fenómeno del tecnoestrés al que anteriormente hemos hecho referencia:

\footnotetext{
${ }^{21}$ Alemán Páez, F.; El derecho de desconexión digital. Una aproximación conceptual, crítica...op. cit.

${ }^{22}$ Rodríguez Escanciano, S.; Poder de control empresarial, sistemas tecnológicos y derechos fundamentales de los trabajadores. Editorial Tirant lo Blanch, $\mathrm{n}^{\circ}$ 1003. 2015. Valencia.
} 


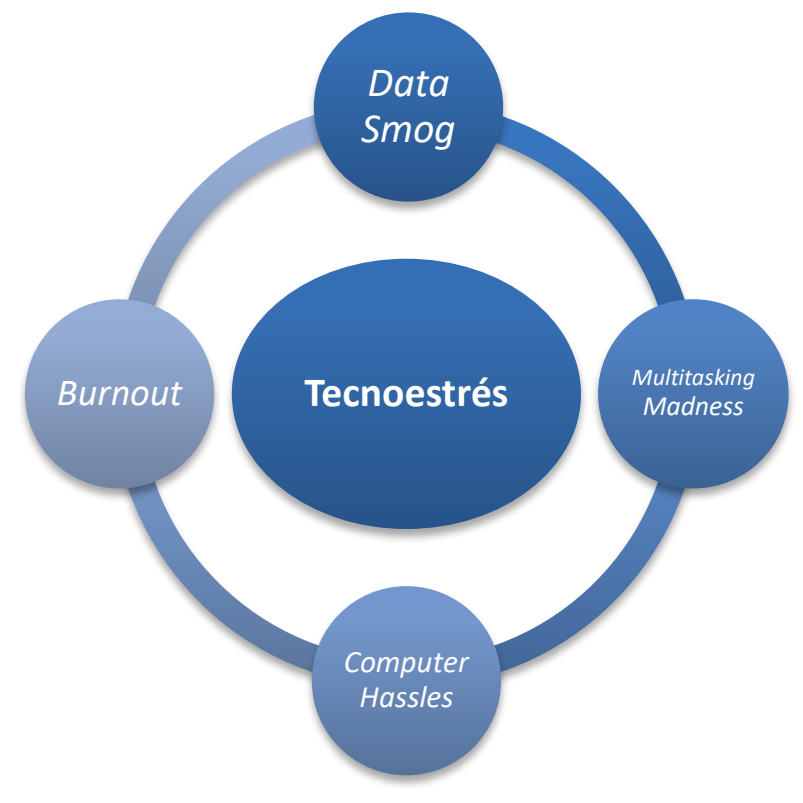

A. Data Smog o síndrome de fatiga informativa. Internet es un sistema que constantemente se encuentra en funcionamiento, siendo considerado como el principal medio de información y de comunicación en todo el mundo. Sin embargo, el exceso de información recibida por parte de los trabajadores mediante la utilización de estos dispositivos electrónicos -correo electrónico, Internet, sistemas de geolocalización, redes sociales, teléfono móvil, etc.-, puede ocasionar situaciones generadoras de estrés en el trabajo, principalmente motivadas por la sobrecarga de información recibida tras la incorporación de las tecnologías en el mismo.

B. MultitaskingMadness o locura multitarea. En ocasiones, los trabajadores pueden no ser capaces de adecuarse al ritmo de trabajo que el uso de las tecnologías requiere para el desarrollo de sus tareas dentro de la relación laboral. En este contexto, se pueden dar lugar a situaciones estresantes derivadas por la dificultad en mantener el ritmo que su puesto exige.

C. ComputerHassles o problemas informáticos. Las tecnologías de la información y de la comunicación pueden dar lugar a una mayor rapidez en el desempeño por parte del trabajador de su actividad laboral; sin embargo, también nos podemos encontrar con sistemas electrónicos caracterizados por su lentitud, por averías en el sistema, virus, informaciones fraudulentas, pérdida de ficheros, etc., que dan lugar a situaciones desesperantes para los trabajadores -principalmente para aquellos problemas que desencadenan pérdidas de datos, documentación o ficheros-.Esta situación origina que los trabajadores tengan que reorganizarse en su puesto con el objetivo de recuperar o repetir aquella información perdida por el uso de las tecnologías, lo cual, indiscutiblemente, provoca un cuadro de situaciones nuevas tecno-estresantes. 
D. Burnout ${ }^{23}$ o síndrome de estar quemado. Esta sintomatología es una de las protagonistas inherentes al tecnoestrés. Fuedenominada por vez primera en 1969 como staffburnout ${ }^{24}$. Posteriormente en la década de los 70 , se acuñó el término que conocemos hoy en día. En 1986, algunos psicólogosnorteamericanos, como C. MASLACH y S. JACKSON, definieron este estado como "un síndrome de cansancio emocional, despersonalización, y una menor realización personal, que se da en aquellos individuos que trabajan en contacto con clientes y usuarios" 25 . Se considera por tanto la forma más importante de estrés laboral al producirse, por el proceso acumulativo del uso de las TIC, un agotamiento emocional de desgaste, fatiga, irritabilidad, y de respuestas negativas hacia uno mismo ${ }^{26}$. Su incorporación al mundo laboral es de aplicación reciente.

En síntesis, los estudios en prevención de riesgos laborales subrayan una amplia gama de perjuicios inherentes al cambio tecnológico. De un lado, la digitalización que se inmiscuye en el mundo laboral de una forma mal canalizada para algunos, elevando los niveles de activación atencional y fisiológica ("tecno-ansiedad"), produciendo cansancio, agotamiento mental y cognitivo ("tecno-fatiga"), e incluso propiciando la compulsión de recibir estímulos conectivos sin solución de continuidad ("tecnoadicción") ${ }^{27}$.

La taxonomía ${ }^{28}$ del tecnoestrés identifica una gran diversidad de trastornos, los cuales han sido señalados por el Observatorio Permanente de Riesgos Psicosociales debido a las consecuencias perjudiciales para la salud de los trabajadores- ${ }^{29}$ :

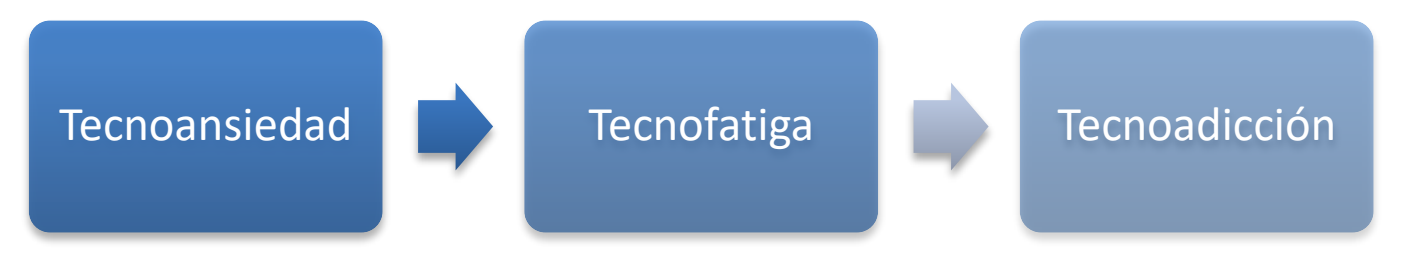

\footnotetext{
${ }^{23}$ Sebastián Calderas, M.L.; Apuntes de Ergonomía. Reflexiones para la práctica de las evaluaciones ergonómicas y psicosociales, Ed. FUNCOP, Sevilla. 2016. Pág. 127 y ss.

${ }^{24} \mathrm{Se}$ utilizó este concepto para referirse al extraño comportamiento que presentaban algunos oficiales de policía de aquella época.

${ }^{26} \mathrm{El}$ Burnout es una experiencia que resulta de situaciones de estrés crónico que se deriva de demandas que exceden los recursos de los empleados y esas demandas se producen con mucha frecuencia en trabajos de servicios. Para más información, puede consultarse el siguiente enlace web: http://www.estreslaboral.info/sindrome-de-burnout.html

${ }^{27}$ Alemán Páez, F.; El derecho de desconexión digital. Una aproximación conceptual,... op. cit.

${ }^{28}$ Alemán Páez, F.; El derecho de desconexión digital. Una aproximación conceptual...op. cit.

${ }^{29}$ Aragüez Valenzuela L.;El uso de las tecnologías como nueva realidad laboral en transformación: el tecnoestrés. Póster presentado al el Congreso Inaugural Cielo, sobre Los actuales cambios sociales y laborales: nuevos retos para el mundo del trabajo. Oporto. 2016
}

e-Revista Internacional de la Protección Social, ISNN 2445-3269. 2017, Vol. II, N 2 
A. Tecnoansiedad: nos referimos a esta situación cuando experimentamos un elevado nivel de actividad fisiológica, así como un incremento de la tensión y el malestar, por el uso de algún sistema o herramienta tecnológica en el trabajo. Es el tipo de tecnoestrés más conocido, donde los trabajadores experimentan altos niveles de activación fisiológica de carácter no placentero y sienten tensión y malestar por el uso, ya sea presente o futuro, de las TIC.

La tecnoansiedad podría ser considerado como un ejemplo claro de tecnofobia, ya que la propia ansiedad de los trabajadores desencadena actitudes escépticas respecto al uso de las tecnologías, ocasionando pensamientos negativos sobre la propia capacidad y competencia de las mismas.

B. Tecnofatiga: se caracteriza por sentimientos de cansancio y agotamiento, tanto de carácter mental como cognitivo, que puede verse incrementado por actitudes de recelo y desconfianza frente a la eficacia de la utilización de las tecnologías en el puesto de trabajo. Este trastorno se encuentra intrínsecamente relacionado con el síndrome de fatiga informativa al que anteriormente hemos hecho referencia, ya que proviene de la sobrecarga de la información que las tecnologías producen a los trabajadores en su uso.

Ciertamente, el desgaste ocupacional y la maximización de los factores (tales como: la iniciativa, la responsabilidad, la autonomía, o la presión por el rendimiento, etc.), se encuentran unidos al sobre-exceso de estímulos informativos y comunicacionales, los cuales desencadenan una fatiga cognitiva y una activación de enfermedades de carácter mental como neuronal.

C. Tecnoadicción: es un fenómeno que se caracteriza por la incontrolable necesidad de hacer frente a un uso continuado, obsesivo y compulsivo de las nuevas tecnologías en todo momento y en todo lugar. Los síntomas en este caso pueden ser: insomnio, depresión, ansiedad, irritabilidad, y soledad. El hecho de pasar mucho tiempo "conectado" a la actividad adictiva (en este caso las tecnologías), hace que se reduzca el tiempo que dedica el adicto a otras actividades tales como: la vida familiar, las relaciones sociales y las aficiones. En el caso de que el trabajador utilice las tecnologías para desarrollar su actividad laboral, puede desencadenar que se produzca una adicción al trabajo por estar constantemente conectado al mismo, dificultando la conciliación de su vida personal y familiar, así como de su salud.

Como podemos observar, queda reflejado que el tecnoestrés es el resultado de un proceso perceptivo de desajustes entre demandas y recursos disponibles, y está caracterizado por dos dimensiones centrales. La primera dimensión hace referencia a los síntomas afectivos o ansiedad relacionada con el alto nivel de activación psicofisiológica del organismo (tecnofilia); y la segunda dimensión recae en el desarrollo de actitudes negativas hacia las $\mathrm{TIC}^{30}$ (tecnofobia).

En síntesis, el tecnoestrés puede dividirse en dos tipos de estrés diferentes en el trabajo.

1. Euestrés: el euestrés o estrés positivo es aquel en el que el organismo respeta la capacidad de respuesta física y psicológica del individuo y actúa como factor motivador que ayuda a superar las dificultades. En este caso, nos encontramos

${ }^{30}$ Salanova Soria, M.;Nuevas tecnologías y nuevos riesgos psicosociales en el trabajo. op. cit.

e-Revista Internacional de la Protección Social, ISNN 2445-3269. 2017, Vol. II, No 2

http://dx.doi.org/10.12795/e-RIPS.2017.i02.12.

Página 181 
con el anteriormente denominado trabajador 3.0-que utiliza de forma positiva la incorporación de las tecnologías con el trabajo y mantiene un equilibrio entre el posible exceso o defecto de la utilización de dichos medios-.

2. Distrés: el distrés o el efecto negativo es aquel en el que la capacidad de la persona resulta insuficiente para hacer frente a la utilización de las tecnologías en el trabajo (tecnofobia)o, por el contrario, utiliza las mismas de forma excesiva creando una modalidad de adicción (tecnofilia).

\section{POSIBLES MEDIDAS PREVENTIVAS DEL TECNOESTRÉS}

La identificación de los factores de riesgo asociados al tecnoestrés y la evaluación de los mismos cuando se han producido de forma inevitable, requiere la determinación de aquellas medidas preventivas necesarias para evitar o minimizar los daños. En este sentido, es preciso citar que algunos países de la Unión Europea han mostrado su preocupación ante la aparición de nuevos riesgos psicosociales como es el tecnoestrés, ya que cada vez un mayor número de empresas ${ }^{31}$ incorporan el uso de las tecnologías de la información y de la comunicación en el desarrollo de su actividad. Así, la exposición del trabajador a diversos riesgos psicosociales que hasta entonces no eran tenidos en cuenta está relacionada con el deterioro de la salud ${ }^{32}$. Sin embargo, todo riesgo debe ir aparejado de una serie de medidas preventivas que eviten o reduzcan las consecuencias negativas del mismo en el trabajo.

De un lado, los recursos laborales de la empresa son totalmente necesarios para reducir el esfuerzo mental y físico de los trabajadores en el desarrollo de su actividad laboral, minimizando por ende los efectos perjudiciales en su salud. La empresa deberá dotar a los trabajadores de los recursos suficientes y necesarios para la utilización de programas informáticos, equipos, plataformas virtuales, etc., con el objetivo de que los trabajadores lleven a cabo un uso de las tecnologías de la información y de la comunicación de forma

\footnotetext{
${ }^{31}$ Según los datos del Instituto Nacional de Estadística, algunos datos en España muestran que el 98,3\% de las empresas de 10 ó más empleados dispone de conexión a Internet en el primer trimestre de 2014.Además, el uso de ordenadores está extendido en la práctica totalidad de estas empresas $(99,2 \%)$. En el 95,3\% de las empresas está implantada la telefonía móvil. Por otro lado, el 75,8\% de las empresas con conexión a Internet dispone de sitio/pagina web. En las de 250 ó más empleados, este porcentaje alcanza el $95,7 \%$.

Estos porcentajes muestran la importancia de las tecnologías en el territorio español, ya que cada vez son más las que intervienen en el uso de las mismas. Por su parte, el 22,9\% de empresas invirtieron en formación en TIC durante 2013. En el caso de las empresas de 250 o más empleados, este porcentaje alcanzó el 59,8\%, seis puntos más que en el año anterior.

Sin embargo, estos datos en relación a la cantidad de empresas que usan las TICS como fuentes de gestión empresarial es bastante bajo, lo cual hace que numerosos trabajadores no cuenten con la formación suficiente para el puesto de trabajo que están desempeñando. En cuanto a las comunicaciones, el 76,5\% de las empresas con menos de 10 empleados usa telefonía móvil, frente al 74,6\% del año anterior. Por su parte, el 21,7\% utiliza otras tecnologías (GPS, TPV,...).

En enero de 2013, el 27\% de las empresas con más de 10 empleados contaban con trabajadores que desempeñaban su actividad fuera de la empresa y se conectaban externamente a ella a través de sistemas TI, 5,2 puntos porcentuales por encima de los valores del año anterior. Continúa la tendencia al alza de los últimos años, pasando del 7\% en diciembre de 2002 a más del triple (27\%) en enero de 2013.

${ }^{32}$ Ortega Ruiz, C., López Ríos, F.; El burnout o síndrome de estar quemado en los profesionales sanitarios: revisión y perspectivas. InternationalJournal of Clinical and HealthPsychology, 2004, Vol. 4, No 1. Almería. Pág. 137-160
}

e-Revista Internacional de la Protección Social, ISNN 2445-3269. 2017, Vol. II, No 2

http://dx.doi.org/10.12795/e-RIPS.2017.i02.12.

Página 182 
razonable y meditada. Para ello, se requiere que la empresa dote a los trabajadores de diversos cursos de formación y aplicabilidad de las tecnologías a su puesto de trabajo, a través del uso de servicios tecnológicos y de software.

De otro lado, el apoyo social a los trabajadores también resulta fundamental como medida preventiva de la posible aparición del tecnoestrés en el trabajo. Esta medida incide a la hora de satisfacer las necesidades de afiliación de los trabajadores, mejorando las condiciones de trabajo adversas. Se debe tratar de evitar o reducir la utilización de las tecnologías de la comunicación para relacionarte con los compañeros en el trabajo, ya que esto puede dar lugar a un aislamiento de la comunicación personal y verbal de entre los mismos. Por ello, se requiere mejorar la comunicación interpersonal de los trabajadores con el objetivo de ayudar a que se sientan parte de un grupo social con proximidad relacional, evitando aquellas condiciones de trabajo que sean amplificadoras de tecnoestrés.

En tercer lugar, resulta fundamental que la empresa informe a los trabajadores de los cambios que va a tener la empresa debido a la incorporación de las nuevas tecnologías, considerando que las mismas van a ser incluidas en el desarrollo de su trabajo, ya sea de forma directa o indirecta. A través de esta información, se les dota alos trabajadores de una mejor adaptación al puesto de trabajo por el hecho de tener un conocimiento previo de aquellos cambios que pueden sufrir en su puesto de trabajo. Esta idea se encuentra igualmente relacionada con la necesidad de implantar programas y planes formativos a los trabajadores, con el fin de que los mismos desarrollen aptitudes, conocimientos y destrezas en la utilización de las tecnologías en su trabajo.

La sobrecarga en el trabajo o el exceso información por el uso de las tecnologías, puede dar lugar a que el trabajador se encuentre colapsado. Así, resultará imprescindible que el empresario desarrolle medidas encaminadas a identificar y evaluar los cambios en los sistemas de trabajo originados por la incorporación de las tecnologías para minimizar o evitar los efectos nocivos de su uso. Para ello, se requiere analizar los factores de riesgo en aquellas situaciones de tecnoestrés que afecten a la salud. Con esta idea, el Observatorio Permanente de Riesgos Psicosociales ha elaborado un estudio donde se agrupan las medidas preventivas a las que anteriormente hemos hecho referencia. En líneas generales son las que a continuación se reflejan:

A. Medidas sobre aspectos técnicos. Se caracterizan fundamentalmente por el diseño adecuado del puesto de trabajo que viene ocupando el trabajador. El principal objetivo por parte del empresario consiste en incorporar las nuevas tecnologías en el trabajo sin que las mismas se entiendan como estresantes, sino que vayan acompañadas por un plan de adaptabilidad atendiendo a las circunstancias o condiciones de los trabajadores.

La introducción al puesto de trabajo del elemento tecnológico debe hacerse teniendo en cuenta como posibles puntos básicos:

- El diseño adaptado o adaptable de las tecnologías a las características personales del trabajador

- El acceso de forma fácil y comprensible a las herramientas tecnológicas. 
- El fomento del trabajador al uso de las tecnologías.

Si por ejemplo un trabajador sufre una serie de dificultades técnicas mediante la utilización de dispositivos o herramientas informáticas que ocasione una sobrecarga de trabajo, ya sea de carácter cuantitativo o de acumulación de tareas, es necesario que la empresa facilite también la correspondiente asistencia al trabajador, y más principalmente cuando su trabajo depende totalmente del uso de las TIC -como ocurre por ejemplo en el caso de los teletrabajadores ${ }^{33}-$.

B. Medidas sobre aspectos organizativos. Los aspectos organizativos de la empresa son un punto clave en el bienestar de los trabajadores.

Para ello, es necesario que el empresario desarrolle las siguientes acciones:

- Mejorar el clima de trabajo, ya que puede desincentivar al trabajador, reduciendo su esfuerzo y aumentando sus ganas de dejar la empresa.

- Fomentar el trabajo en equipo.

- Dotar a los trabajadores de un mayor grado de autonomía mediante la creación de sistemas eficaces de comunicación.

- Rediseñar los puestos de trabajo para una mejora en la adaptabilidad;

- Informar a los trabajadores de la incorporación de las tecnologías de la información y de la comunicación en su trabajo, para así evitar sentimientos de miedo o rechazo.

- Adecuar la cantidad y la complejidad del trabajo, los ritmos de trabajo y la cantidad de tiempo necesario para desarrollar la actividad laboral en el puesto de trabajo de cada trabajador, son acciones preventivas imprescindibles para el empresario.

- Incentivar por parte del empresario la colaboración y cooperación entre compañeros de trabajo, ya que dicho apoyo informático promovido por la transmisión de conocimientos puede prevenir la aparición de situaciones estresantes.

- Crear un espacio de interacción e intercambio de información entre los trabajadores-mayoritariamente cuando se encuentran en diferentes edificios u oficinas- a través de canales de comunicación virtual (foros, chats, video llamadas, etc.).

C. Medidas sobre el trabajador. El trabajador debe mostrar una actitud positiva ante la incorporación de las tecnologías de la información y de la comunicación en su puesto de trabajo. Para ello, el empresario debe fomentar la participación de los trabajadores en la elección de las tecnologías e incorporar una serie de encuestas de satisfacción con opciones de recomendación.

\footnotetext{
33 Así especialmente viene señalado en el Acuerdo Marco sobre Teletrabajo (art. 7). Disponible en el siguiente enlace: http://www.uned.ac.cr/viplan/images/acuerdo-marco-europeo-sobre-teletrabajo.pdf
} 
Para desarrollar estas medidas en el trabajo, se considera imprescindible la inversión en una formación de calidad, diseñando cursos específicos en función de las necesidades de los trabajadores, ajustando sus conocimientos, capacidades y perfiles, y evitando que se consideren ineficaces, ya que cuando una persona está más formada en aquello que realiza, siente menos miedo ante situaciones no controladas por ellos mismos y, a su vez, tendrá una mayor adaptabilidad a los cambios que puedan producirse por las tecnologías.

GRAU GUMBAU, junto con SALANOVA SORIA y PEIRÓ SILLA ${ }^{34}$, realizaron un estudio cuyo fundamento versa en considerar el control, es decir, la autosuficiencia, como elemento clave del proceso de estrés. Entendían que la mera exposición a estresores con control por parte del sujeto, no tiene efectos adversos; mientras que si, por otro lado, la exposición a los estresores se realizase sin control, si pueden producirse efectos indeseados o nocivos para él. Textualmente señalan que "analizar el rol modulador de la auto eficacia generalizada y la auto eficacia profesional en las relaciones entre determinadas características del trabajo -de la tarea y del ambiente social-" puede "percibirse como estresores (autonomía en el puesto, clima de apoyo social, rutina en las tareas y conflicto de rol), y sus consecuencias (burnout agotamiento emocional y cinismo-, satisfacción laboral y compromiso organizacional)".

Los resultados obtenidos en relación con la modulación del burnout, son importantes para el diseño de estrategias orientadas a su prevención a partir del rediseño del trabajo. Quizá una forma de prevenirlo podría ser disminuyendo las demandas para prevenir el cinismo y aumentar los recursos (autonomía ${ }^{35}$ ) para prevenir el agotamiento emocional.

Lo que se debe poner de manifiesto con este estudio es que el estrés laboral está presente en las organizaciones estudiadas y muchas veces aparece como inevitable -y más concretamente cuando las situaciones estresantes son difíciles de controlar por parte de la organización empresarial ${ }^{36}$. En este sentido, mencionan que una estrategia de prevención adecuada podría ser mejorar la autoeficacia de los trabajadores mediante la formación. Sin embargo, lo cierto es que estas situaciones tecnoestresantes son totalmente evaluables, al menos la mayoría de ellas, por parte de la empresa. Esto da lugar a que el empresario tenga la obligación, como cualquier otra situación de riesgo en la salud de los trabajadores, de responder ante los efectos negativos que se produzcan por la utilización de las tecnologías en el trabajo.

Las medidas preventivas citadas pueden resultar ser los primeros pasos para minimizar los daños que la exposición del tecnoestrés puede repercutir en la salud de los trabajadores. Pero a su vez, se considera de suma importancia evaluar por parte del empresario los posibles riesgos en la salud tras la incorporación de las tecnologías en el

\footnotetext{
${ }^{34}$ Grau Giumbau, R.M., Salanova Soria, M., Peiró Silla, J.M.;Efectos moduladores de la autoeficiencia en el estrés. Dialnet, Vol. 18, Nº 2000, págs. 57-75. Disponible en el siguiente enlace: http://www.apuntesdepsicologia.es/index.php/revista/article/viewFile/414/334.

${ }^{35}$ Niveles altos de autonomía en el trabajo no están asociados con un mayor bienestar psicológico de forma directa, sino que esto depende de los niveles de autoeficacia profesional. Así, para trabajadores con baja autoeficacia profesional, una mayor autonomía (que generalmente se relaciona con una mayor responsabilidad y reto profesional) se asocia con un mayor agotamiento emocional. Estos resultados tienen claras implicaciones en el rediseño de trabajo y en la adecuación

${ }^{36}$ Grau Giumbau, R.M., Salanova Soria, M., Peiró Silla, J.M.; Efectos moduladores de la autoeficiencia... op. cit.
}

e-Revista Internacional de la Protección Social, ISNN 2445-3269. 2017, Vol. II, No 2

http://dx.doi.org/10.12795/e-RIPS.2017.i02.12.

Página 185 
puesto de trabajo, para así adoptar diversas medidas preventivas adecuadas a cada puesto de trabajo en concreto, ajustando lo máximo posible cada puesto con las capacidades de los trabajadores.

\section{AUSENCIA DE REGULACIÓN ESPECÍFICA Y ANÁLISIS DEL DERECHO A DESCONECTARSE}

Los empresarios españoles realizan considerables inversiones en adaptar los recursos productivos al entorno empresarial que es cada vez más competitivo ${ }^{37}$. Así, la utilización de cualquier medio de carácter conectivo - como pueden ser los correos electrónicos, los móviles, las tabletas, los teléfonos inteligentes, los iPads, las redes sociales, etc.-, trasciende de los lugares donde el trabajador desarrolla su actividad laboral normalmente, es decir, las empresas, los centros o los diferentes puestos ocupacionales de trabajo. De este modo, la unidad locativa, donde hasta entonces venía desarrollando su trabajo, se convierte en un lugar movible y multifuncional.Además de ello, el tiempo de trabajo se considera atemporal, debido a que la sociedad de la información y de la comunicación mantiene una conectividad cuasi permanente mediante diversos dispositivos digitales, los cuales facilitan y propician la dilución, la flexibilidad de la jornada de trabajo y los tiempos de ocio y de recuperación.

Sin embargo, y con independencia de que la sociedad avance en el uso de las nuevas tecnologías en el trabajo, nos encontramos con una gran deficiencia legislativa en esta materia, por lo que el problema que se plantea es que, a pesar de que en España exista cada vez más empresas que aplican el uso de las TIC en su gestión empresarial con el objetivo de aumentar su productividad, la escasa (por no decir ausente) regulación específica en la materia, resulta latente y preocupante, no sólo a nivel nacional, sino también en el ámbito comunitario.

A nivel nacional y como regla excepcional, nos podemos encontrar con la Ley Orgánica 5/1992, de 29 de octubre, de Regulación del Tratamiento Automatizado de Datos de Carácter Personal (LORTAD), que posteriormente fue sustituida por la Ley Orgánica 15/1999, de 13 de diciembre, de Protección de Datos de Carácter Personal, que supone la adaptación de la Directiva 95/46/CE, de 24 de octubre de 1995. La cual muy próximamente será sustituida por el Reglamento (UE) 2016/679 del Parlamento europeo y del Consejo de 27 de abril de 2016. A su vez, completan el elenco con la Ley 34/2002, de 11 de julio, de servicios de la Sociedad de la Información y del Comercio Electrónico $\mathrm{y}$ algunas otras escasas directivas comunitarias ${ }^{38}$.

El desierto de regulación legal específica obliga a los operadores jurídicos y a los tribunales a realizar esfuerzos aplicativos de las normas generales al ámbito laboral. El

\footnotetext{
${ }^{37}$ Alfaro de Prado Sagrega, A.; Nuevas tecnologías y nuevos riesgos laborales: estrés y tecnoestrés. Revista Digital de Seguridad y Salud en el trabajo de la Universidad de Huelva, $n^{\circ}$ 1. 2008. Pág. 16

${ }^{38}$ Directiva 95/46/CE del Parlamento Europeo y del Consejo de 24 de julio de 1995, relativa a la protección de las personas físicas en lo que respecta al tratamiento de datos personales y a la libre circulación de estos datos; Directiva 97/66/CE del Parlamento Europeo y del Consejo, de 15 de diciembre de 1997, relativa al tratamiento de los datos personales y a la protección a la intimidad en el sector de las telecomunicaciones; Directiva 2002/58/CE del Parlamento Europeo y del Consejo, de 12 de julio, relativa al tratamiento de los datos personales y a la protección de la intimidad en el sector de las telecomunicaciones (Directiva sobre la privacidad y las comunicaciones electrónicas).
}

e-Revista Internacional de la Protección Social, ISNN 2445-3269. 2017, Vol. II, No 2

http://dx.doi.org/10.12795/e-RIPS.2017.i02.12.

Página 186 
hecho causante de esta situación hace referencia a que el empresario pueda actuar de forma arbitraria, aprovechándose de la falta de legislación y dando lugar a situaciones de abuso a los trabajadores.

Uno de los aspectos más controvertidos que afectan a la forma de organizar el trabajo es el relativo al tiempo a la hora de desempeñar el trabajador su jornada de trabajo, donde, a través de la utilización de medios telemáticos, se ha visto notablemente flexibilizado por una disponibilidad constante e hiperconectividad en cualquier momento e incluso fuera del horario de trabajo de los trabajadores. Esto origina situaciones negativas que directamente recaen en la salud de los trabajadores -al ser generadoras de tecnoestrés- $\mathrm{y}$, a su vez, en la conciliación de su vida personal y familiar por encontrarse conectados al entorno de trabajo de forma interrumpida.

Bajo esta premisa, es necesario decir que los tribunales en España han tenido que recordar a los empresarios lo que se consideran, por poner un ejemplo, horas extraordinarias $^{39}$, concepto que pareció olvidado tras la aparición de los nuevos instrumentos informáticos y electrónicos de la información y de la comunicación, debido al hecho de que se consideraba que, mediante el uso de las tecnologías, se encontraba efectuando el trabajador su trabajo incluso fuera de su jornada laboral. En un principio, y aunque parezca descabellado, la utilización de las tecnologías en el desarrollo de la actividad laboral fuera del horario de trabajo, no se entendía como horas extraordinarias o al menos en la práctica (Sentencia del Tribunal Superior de Justicia de Castilla y León $^{40}$ ), por lo que formalmente no eran ni abonadas económicamente ni compensadas.

A partir de esta sentencia, estas horas "virtuales" y fuera de la jornada laboral, se entienden como horas "reales", mereciendo la consideración de horas extraordinarias. Es cierto que el debate sobre la hora exacta en la que debe finalizar la jornada laboral continúa, pero al menos parece que los tribunales tratan de solventar determinadas situaciones de abuso del poder empresarial por la incorporación de las tecnologías ${ }^{41}$ en el

\footnotetext{
${ }^{39}$ Real Decreto Legislativo 2/2015, de 23 de octubre, por el que se aprueba el texto refundido de la Ley del Estatuto de los Trabajadores señala en su artículo 35 lo que se entiende por horas extraordinarias: 1. Tendrán la consideración de horas extraordinarias aquellas horas de trabajo que se realicen sobre la duración máxima de la jornada ordinaria de trabajo, fijada de acuerdo con el artículo anterior. Mediante convenio colectivo o, en su defecto, contrato individual, se optará entre abonar las horas extraordinarias en la cuantía que se fije, que en ningún caso podrá ser inferior al valor de la hora ordinaria, o compensarlas por tiempos equivalentes de descanso retribuido. En ausencia de pacto al respecto, se entenderá que las horas extraordinarias realizadas deberán ser compensadas mediante descanso dentro de los cuatro meses siguientes a su realización.

40 STSJ de Castilla y León 198/2016, 3 de febrero de 2016 (Rec. № 2229/15). "Solamente si la empresa ha establecido pautas claras sobre el tiempo de trabajo respetuosas con la regulación legal y convencional sobre la jornada y descansos y si además establece, de acuerdo con el trabajador, instrumentos de declaración y control del tiempo de trabajo a distancia o en el domicilio, sería posible admitir que una conducta del trabajador en el interior de su domicilio en vulneración de dichas pautas y omitiendo los instrumentos de control empresarial pudiera dar lugar a exceptuar el pago de las correspondientes horas y su computo como tiempo de trabajo. Pero en ausencia de estas pautas y criterios y de unos mínimos instrumentos de control, no existe razón para no computar las horas dedicadas al trabajo como jornada ni el pago de los excesos como horas extraordinarias".

${ }^{41}$ En una encuesta realizada el año 2010 a los trabajadores del Huffington Post para conocer sus hábitos de trabajo, se encontró que el $60 \%$ reconocía que sólo se desconectan completamente del correo electrónico dos horas al día y el $20 \%$, menos de media hora.

Otra encuesta realizado por Cisco Systems revela que el $45 \%$ de los empleados con acceso externo al sistema informático de la empresa trabaja entre dos y tres horas más al día. Una cuarta parte de ellos
}

e-Revista Internacional de la Protección Social, ISNN 2445-3269. 2017, Vol. II, No 2

http://dx.doi.org/10.12795/e-RIPS.2017.i02.12.

Página 187 
trabajo que, hasta día de hoy, continúan quedando fuera del marco legislativo.

Los derechos de desconexión representan una réplica frente a la dilución de los códigos de espacio y tiempo antes descrita. El ordenamiento francés, a diferencia del español, da apertura a un "nuevo derecho" que garantiza las condiciones de descanso, reposo y aislamiento de los trabajadores -salvo casos excepcionalmente justificados-; pero también trata de preservar los derechos de conciliación de la vida personal y familiar en planos paralelos de protección jurídica y la salud de los mismos. De esta forma, se ha regulado por el ordenamiento francés y muy recientemente el conocido: droit á la déconnexion ${ }^{42}$ o derecho a desconectarse (categoría ius-tecnológica), en vigor desde el uno de enero del 2017, que enlaza con fines protectores de la salud, el medio ecológico de trabajo y los derechos de conciliación ${ }^{43}$.

La regulación de este derecho resulta un gran avance en el proceso positivizador de la digitalización laboral, ya que "se instituye entonces un trinomio protector que, por un lado, anude la salvaguarda de los riesgos tecnológicos, la salud laboral y los derechos de conciliación, empero, proyectando a un mismo tiempo una relación con-causal entre dichos anclajes institucionales" ${ }^{\prime 4}$. En este análisis se considera el derecho a desconectarse como una medida positiva para paliar el tecnoestrés laboral, encaminada además a un inicio de nuevos cambios para adaptar el derecho del trabajo clásico a la nueva era digital de la información y de las comunicaciones.

El ministro francés de educación nacional, BENOÎTHAMON, señaló textualmente: "Los estudios muestran que hay mucho mas estrés relacionado con el trabajo hoy en día que antes y que éste es constante. Los empleados dejan físicamente la oficina pero no dejan su trabajo. Ellos permanecen unidos por una especie de lazo electrónico, como un perro. Los textos, los mensajes y los correos electrónicos colonizan la vida del individuo hasta el punto en que él o ella finamente se rompe". A raíz de ello, adoptar como medida paliativa clave del tecnoestrés el derecho a desconectarse resulta, sin lugar a dudas, un acierto ${ }^{45}$, ya que se muestra la preocupación por parte ordenamiento jurídico francés de la incontrolable intromisión del trabajo en la vida privada de cada empleado, haciéndose insostenible si no se regula esta cuestión por precepto legal. Este derecho es considerado como una forma de concienciar a la sociedad -y más concretamente a los trabajadores-de que hay que poner límites entre la vida privada y el trabajo, para evitar la aparición de posibles riesgos psicosociales.

Concretamente en el ordenamiento francés goza de un gran protagonismo la

trabaja hasta cuatro horas extras. Un $26 \%$ de los empleados que trabajan con TICS cree que el uso de Internet y del móvil aumenta su horario laboral y que esa disponibilidad permanente le genera estrés

${ }^{42}$ Ley 2016-1088, de 8 de agostodu 8 août 2016 relativeautravail, à la modernisation du dialogue social et à la sécurisation des parcoursprofessionnels. Para visualizar su contenido: JournalOfficiel de la RépubliqueFrancaise (JORF) $\quad \mathrm{N}^{\mathrm{o}} 184 \mathrm{du} \quad 9$ aoút 2016 (texte $\mathrm{N}^{\mathrm{o}}$ 3): http://travailemploi.gouv.fr/IMG/pdf/loi_no2016-1088_du_8_aout_2016_version_initiale.pdf.

${ }^{43}$ Alemán Páez, F.; El derecho de desconexión digital. Una aproximación conceptual, ... op. cit.

${ }^{44}$ Alemán Páez, F.; El derecho de desconexión digital. Una aproximación conceptual,... op. cit.

${ }^{45}$ Muchos otros investigadores, tales como Jon Whittle, señalan que la idea del derecho a desconectarse no es adecuada por el motivo de que los trabajadores se pueden encontrar sobrecargados de trabajo y sentirse abrumados por una avalancha de mensajes al volver por ejemplo de las vacaciones o de unos días de descanso y desconexión. Sin embargo, lo que es cierto es que permanecer conectados constantemente fuera del horario de trabajo y que no se regule esta situación es insostenible.

e-Revista Internacional de la Protección Social, ISNN 2445-3269. 2017, Vol. II, No 2

http://dx.doi.org/10.12795/e-RIPS.2017.i02.12.

Página 188 
negociación colectiva debido a que ha dejado en manos de los representantes e intervinientes su contenido sustancial, mediando un sistema de acuerdos. El objetivo de dejar en manos de la negociación la regulación de este derecho es debido a que, de esta manera, se puede definir de mejor modo el ejercicio del derecho a la desconexión en cada puesto de trabajo concreto, fomentando y haciendo prevalecer las acciones de formación -que a lo largo de este estudio hemos observado que son sumamente importante- y de sensibilización respecto al uso de forma razonable y adecuada de los dispositivos digitales en el trabajo.

A pesar de esta escueta legislación en lo que a las nuevas tecnologías de la información y de la comunicación se refiere, la regulación del derecho a desconectarse en el ámbito laboral potencia la negociación de las empresas en las políticas formales y limita la propagación del trabajo y el exceso de conectividad de los trabajadores, mejorando la conciliación de la vida personal, familiar y laboral de los trabajadores, así como también de su salud.

\section{CONCLUSIONES}

La incorporación de las nuevas tecnologías de la información y de la comunicación al mundo empresarial y laboral, ha dado lugar a que los trabajadores se enfrenten al desafío de una nueva necesidad de adaptación, la cual, a veces, resulta insuficiente por parte de los mismos (capacidad en defecto), dando lugar a situaciones tecnoestresantes (estrés motivado por el uso de las nuevas tecnologías) en el trabajo. Sin embargo, el uso en exceso o el abuso en el manejo de las tecnologías en el trabajo, también puede tener efectos nocivos para el trabajador, pudiendo llegar a ser considerados como tecnoadictos. Esto puede provocar que el tiempo de descanso, de ocio, de relacionarse con otras personas, etc., se encuentre dificultado por estar los trabajadores constantemente "conectados" al mundo laboral, dificultando la conciliación de su vida personal y familiar con el trabajo, y teniendo implicaciones perjudiciales en la salud.

En este contexto, han aparecido nuevos riesgos psicosociales que afectan directamente a la salud de los trabajadores, como es el caso del tecnoestrés. Esta situación debe ser directamente evaluada por los empresarios para proceder a la creación de planes de prevención de riesgos laborales. Sin embargo, la adopción de medidas preventivas ante situaciones tecnoestresantes no es tarea fácil, y aún más cuando estamos hablando de un teletrabajador que, por ejemplo, tenga su centro de trabajo en su vivienda; sin embargo, por parte del empresario se debe analizar la adaptabilidad de los trabajadores en su puesto de trabajo y las posibles implicaciones negativas del uso de las TIC en su salud (donde la formación goza de gran importancia).

De otro lado, la falta de regulación en la materia, debido a que la Directiva de Tiempo de Trabajo 2003/88/EC no regula nada específico con respecto a los trabajadores móviles ni tan siquiera referente a la teledisponibilidad, parece que da lugar a que toda forma de trabajo considerada diferente a la tradicional, debe quedar circunscrita a los convenios colectivos o a los acuerdos celebrados entre los interlocutores sociales ${ }^{46}$,

\footnotetext{
${ }^{46}$ Manzano Santamaría, N.; Las tecnologías de la comunicación y la información (TIC's) y las nuevas formas de organización del trabajo: factores psicosociales de riesgo.op. cit
} 
originando un escenario poco favorable para los trabajadores ${ }^{47}$.Así, se hace necesario que por parte del ordenamiento jurídico español se potencie la idea de regular este nuevo escenario laboral y tecnológico para adaptar el derecho del trabajo clásico a la nueva era digitalizada -como ocurre con la nueva "económica colaborativa ${ }^{48 "}$ ".

Un elemento clave, que a su vez tiene cabida en España como consecuencia del encontrarse estrechamente relacionado con el ordenamiento francés, es el "derecho a desconectarse". Con esta medida, según hemos visto a lo largo del estudio, se ha abierto una etapa de positivación jurídica que tiene como objetivo la minimización del impacto tecnológico en el trabajador en cuanto a persona, garantizando su descanso, su reposo y, por supuesto, su desconexión cuando finaliza la jornada laboral.

Este tipo de medidas deben ser incluidas en el resto de países de la Unión Europea, con el fin de potenciar la negociación empresarial en la adopción de planes que regulen los nuevos riesgos psicosociales y cambien la cultura tradicional que es considerada con un papel protagonista en los tiempo actuales. Así, se contribuye a crear una mayor y adecuada conciencia empresarial sobre el uso de las nuevas tecnologías en el trabajo, no entendiendo las mismas como herramienta de uso de poder frente a los trabajadores (abusando en su utilización, o quebrantando la privacidad de los trabajadores), sino respetando sus derechos. Sólo de esta forma dejaremos de ver las TIC como una amenaza y tendrán la consideración de clave de éxito a futuro.

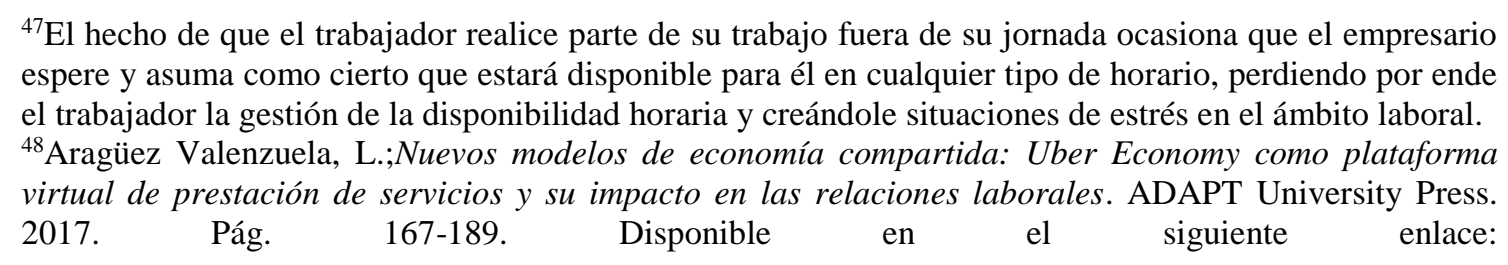
https://dialnet.unirioja.es/servlet/articulo?codigo $=5987918$. 